\title{
The unilateral field advantage in repetition detection: Effects of perceptual grouping and task demands
}

\author{
Matthew T. Hayes, Khena M. Swallow, and Yuhong V. Jiang \\ University of Minnesota, Twin Cities, Minneapolis, Minnesota
}

\begin{abstract}
This study examines interhemispheric interactions in detecting objects that are simultaneously repeated in an array of objects. Previous studies have shown that presenting two identical objects to a single hemifield speeds up repetition detection. This unilateral field advantage (UFA) is often attributed to the relatively low-level processing demands for detecting a perceptual repetition, and more specifically, to more efficient perceptual grouping processes within a hemisphere than between hemispheres. To directly examine the impact of perceptual grouping and task demands on interhemispheric interactions, we asked participants to judge whether four items, one presented in each visual quadrant, were all different, or whether any two were the same, along an instructed dimension. We found that in comparison with the UFA for identical objects, the UFA for repetition detection in accuracy was similar or greater when the matching objects were not perceptually identical and differed in color, size, or viewpoint. Thus, decreasing grouping strength and increasing computational complexity did not reduce the UFA. Results are interpreted in terms of the callosal degradation account of the UFA.
\end{abstract}

From grocery shopping to interpreting scientific graphs, many activities require humans to identify visual objects and compare them with neighboring objects. One simplified laboratory task that taps into the identification and comparison processes is the "repetition-detection" task (Cavanagh \& Parkman, 1972), in which objects are presented simultaneously in an array for participants to detect the repetition of two identical objects. When there are relatively few objects arranged symmetrically in the field (Figure 1), repetition detection is faster when the repeated objects are presented within a single visual hemifield rather than in both visual hemifields (Butcher \& Cavanagh, 2008; Weissman, Banich, \& Puente, 2000). ${ }^{1}$ This unilateral field advantage (UFA) has been attributed to the engagement of relatively simple, low-level perceptual matching processes (Banich \& Belger, 1990) that are more efficiently performed within a hemisphere than across hemispheres (Butcher \& Cavanagh, 2008).

This study examines whether perceptual grouping can provide a full account of the UFA by manipulating perceptual grouping strength and task demands. We adopt the four-element symmetrical display ${ }^{2}$ used by Butcher and Cavanagh (2008), which yields a robust UFA for repetition detection of colors, letters, orientations, circle sizes, and motion directions. The unique aspect of our study is that we ask participants to detect the repetition of a spe- cific stimulus property while varying other properties of the stimuli. In Experiment 1, participants were asked to detect a repetition in either the color or the letterform of letters. The repeated letters could be identical (e.g., two red "A"s) or differ along the irrelevant dimension (e.g., a red "A" and a green "A," in the case of the letterform task). Perceptual grouping strength is reduced when the repeated letters differ in one dimension. In addition, the demand for selecting the relevant dimension for comparison is increased. If the UFA is weakened by decreased perceptual grouping or by increased task demand, then it should be weaker when the repeated letters differ in color than when they are identical. Experiment 2 used a similar logic to test whether the UFA in detecting repeated shapes is weakened when the repeated objects differ in size or viewpoint. Experiment 3 focused on one-dimensional objects (e.g., color patches) but manipulated perceptual grouping and task demands by making the repeated colors distinctive or similar to unrepeated colors. We examined whether simple predictions derived from accounts based on perceptual grouping or task complexity would hold in these experiments. To avoid multiple eye fixations, we used briefly presented visual displays and focused on response accuracy. In unreported data, we confirmed these results in speeded response time (RT) tasks with unlimited display durations.

K.M. Swallow, khena.swallow@gmail.com, or Y.V. Jiang, yuhong.jiang@gmail.com 


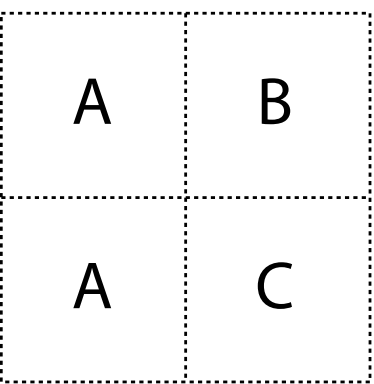

faster

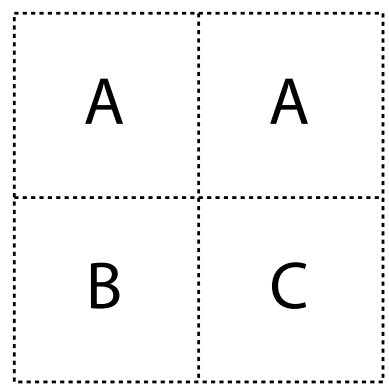

slower

Figure 1. Detecting the exact repetition of letters is faster when the repeated letters are within hemisphere (left) rather than between hemispheres (right), a unilateral field advantage.

\section{EXPERIMENT 1}

\section{Method}

Participants. Participants (18-35 years old) were recruited from the University of Minnesota. They possessed normal color vision and normal or corrected-to-normal visual acuity.

Eight participants (mean age, 25 years; 5 females) completed Experiment $1 \mathrm{~A}$ and 8 others (mean age, 23 years; 6 females) completed Experiment 1B. All but 1 male participant (in Experiment 1A) were right-handed.

Equipment. Participants were tested individually in a normally lit interior room. They sat unrestrained at approximately $57 \mathrm{~cm}$ from a 19-in. CRT monitor. The experiments were programmed in MacProbe (Hunt, 1994).

Stimuli. The items were eight uppercase letters ("A," "B," "C," "S," "E," "F," " $\mathrm{H}$," and "K") in Helvetica font $\left(1.5^{\circ} \times 1.5^{\circ}\right)$ printed in eight distinctive colors (red, green, blue, yellow, purple, cyan, white, or black) against a gray background. Each display consisted of four letters, one in each visual quadrant. The letters were presented simultaneously and were horizontally and vertically aligned (see Figure 1). The center of each item was $3.5^{\circ}$ away from fixation.

Task and Procedure. On each trial, a white fixation $\operatorname{dot}\left(0.2^{\circ} \times\right.$ $0.2^{\circ}$ ) was presented for $500 \mathrm{msec}$, followed by the display of four items presented for $160 \mathrm{msec}$ and then erased. Participants were asked to fixate at the center and judge whether the four items were all unique in an instructed dimension (e.g., color), or whether any two items were the same in that dimension. Participants were told to respond as accurately as possible by pressing the " $\mathrm{d}$ " or " $\mathrm{s}$ " key. Immediately after each response, accuracy feedback in the form of a happy or sad face icon was displayed for $200 \mathrm{msec}$ at fixation. The next trial commenced $1 \mathrm{sec}$ after the feedback.

Design. Each participant completed six blocks of trials. There were 96 trials per block in Experiment 1A and 64 trials per block in Experiment 1B. Participants were instructed to attend to color on three blocks and to letterform on the other three blocks. The blocks were presented in a random order. Participants were encouraged to take a break after each block, during which instructions for the next block's task were shown. In each block, half of the trials were unique trials, on which all four items were unique in the relevant dimension. The value of the irrelevant dimension was randomly selected for each item in unique trials and could have repeated. For the other half of the trials (repetition trials), two of the items were the same in the relevant dimension. For these two items, the irrelevant dimension could also match ( $50 \%$ of the time) or differ ( $50 \%$ of the time). In Experiment 1A, the two items that repeated in the relevant dimension could be arrayed diagonally (main diagonal or antidiagonal, each one sixth of the time), bilaterally (both were in the upper visual field or lower visual field, each one sixth of the time), or unilaterally (both were in the left or right visual field, each one sixth of the time). In Experiment 1B, the diagonal-repetition trials were eliminated and participants were instructed that the repeated items would be adjacent to each other, reducing the number of possible across-hemifield comparisons. The color and letterform of the remaining two items were randomly selected provided that they did not create a repetition in the relevant dimension.

\section{Results and Discussion}

To examine the UFA in repetition detection, we concentrate on the comparison of bilateral and unilateral repetition trials. However, we note that in Experiments 1A, 2A, and $3 \mathrm{~A}$, responses were less accurate and slower for diagonally repeated trials than for bilaterally or unilaterally repeated trials, possibly because the diagonal objects were farther apart. Because the displays were presented briefly and responses were unspeeded, our analyses focused on accuracy (Figure 2). The RTs from correct trials are listed in the Appendix.

A repeated measures ANOVA on experiment as a between-subjects variable, and task (color or letter), irrelevant dimension (repeated or nonrepeated), and visual field arrangement (bilateral or unilateral targets) as within-subjects variables revealed a main effect of task, because accuracy was higher in the color task than in the letter task $[F(1,14)=38.84, p<.001]$. A significant main effect of repetitions in the irrelevant dimension $[F(1,14)=58.68, p<.001]$ indicated that mismatches in the irrelevant dimension reduced accuracy. A significant interaction between task and irrelevant dimension indicated that reductions in accuracy due to mismatches in the irrelevant dimension were greater when color was irrelevant than when letterform was irrelevant $[F(1,14)=$ 27.06, $p<.001]$. Therefore, mismatches in color were more difficult to ignore than were mismatches in letterform. These results held for both Experiments 1A and 1B, because none of the factors mentioned above showed significant interactions with experiment [largest $F(1,14)=$ $2.17, p>.16]$.

Notably, the main effect of visual field arrangement was significant $[F(1,14)=108.26, p<.001]$, indicating a UFA. This effect was stronger when participants attended to letterform rather than color, leading to a significant interaction between task and visual field arrangement $[F(1,14)=7.43, p<.016]$. However, there was no evidence that the UFA was smaller when items that matched on the relevant dimension did not match on the irrelevant dimension. To the contrary, the UFA was significantly larger when the irrelevant dimension did not repeat, leading to a significant interaction between visual field arrangement and irrelevant dimension repetition $[F(1,14)=$ $6.24, p<.026]$. The three-way interaction between task (color or letterform), visual field, and irrelevant dimension was insignificant $[F(1,14)=3.12, p=.10]$.

Although the pattern of data was similar across experiments, the UFA was significantly greater in Experiment 1B than in Experiment 1A $[F(1,14)=6.71, p<$ $.021]$ (the UFA was significant in both experiments, $p$ s $<$ .001 ). Therefore, the UFA was not reduced when diago- 

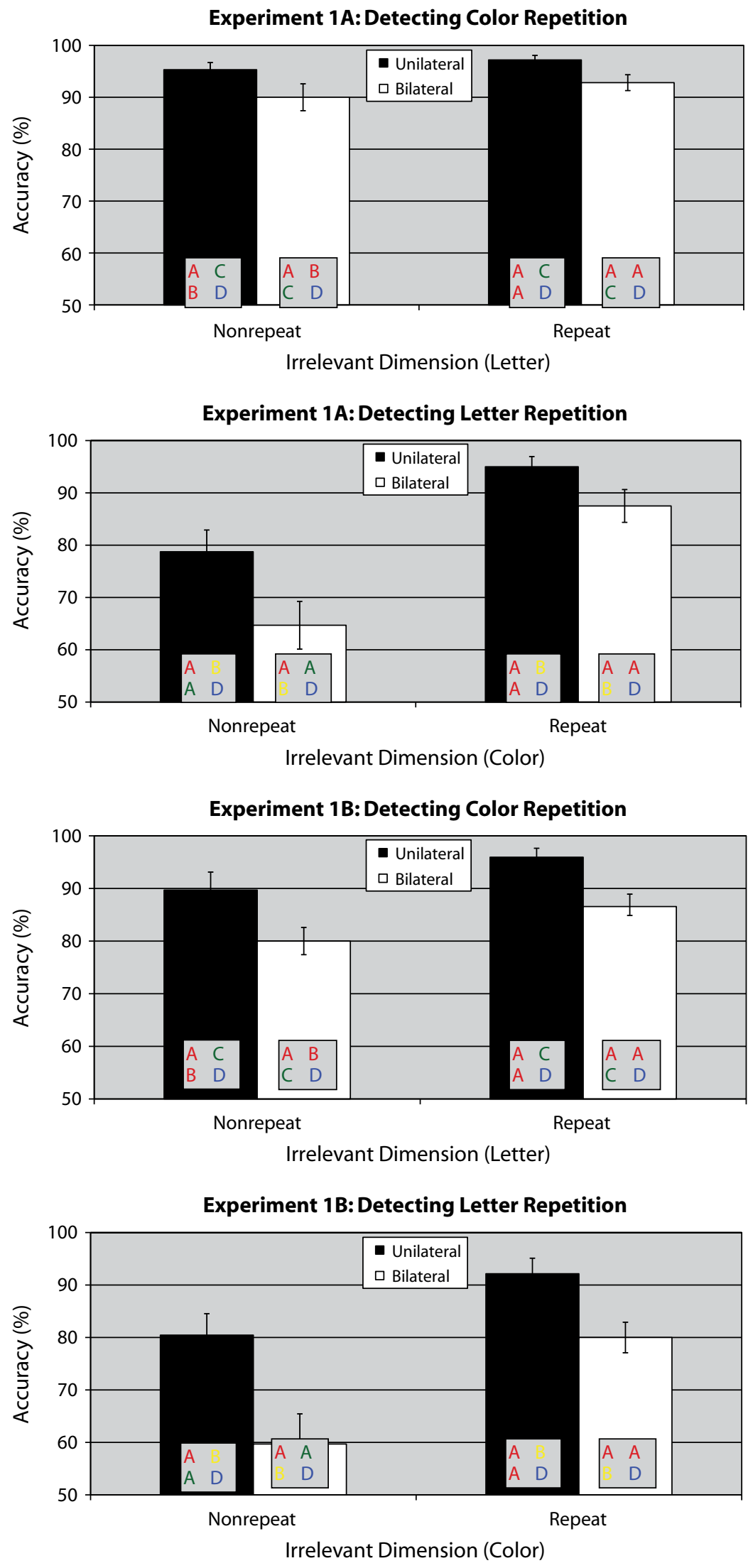

Figure 2. Accuracy results from Experiment 1. Participants attended to either color or letter. Error bars show $\pm 1 S E$ across participants. 
nal trials were excluded, arguing against the notion that it can be attributed to the number of bilateral versus unilateral repetition comparisons. The accuracy data could not be accounted for by speed-accuracy trade-off (see the Appendix).

The data from this experiment provided no evidence that the UFA was smaller when grouping cues were weaker (i.e., the repeated targets differed in the irrelevant dimension). In the mismatch condition, additional computations were needed to segregate and ignore the irrelevant dimension (MacLeod, 1991; Weissman \& Banich, 1999), but this factor also did not attenuate the UFA.

\section{EXPERIMENT 2}

This experiment used everyday objects that were of different sizes or were viewed from different viewpoints. Participants were told to focus on shape and ignore differences in size and viewpoint. The repeated targets could be identical or different in size (one was twice as large as the other) or viewpoint (one was rotated by at least $90^{\circ}$ in 3-D). Previous studies have shown that differences in viewpoint or size slow down perceptual comparisons (Vickery, King, \& Jiang, 2005) and object recognition (Peissig \& Tarr, 2007). To detect repetitions in object shape, some transformation must be computed for objects that differ in size or viewpoint. Additionally, perceptual similarity between repeated targets is reduced when they are mismatched in size or viewpoint. We examined whether the UFA in detecting repeated shapes would be attenuated in these conditions.

\section{Method}

Participants. Nine participants (mean age, 25 years; 7 females) completed Experiment 2A and 9 others (mean age, 22 years; 8 females) completed Experiment 2B. All participants reported being right-handed.

Stimuli. We selected 20 distinctive objects from an online database and converted them into grayscale images. There were two viewpoints for each object that differed by at least $90^{\circ}$ in $3-\mathrm{D}$. On each trial we randomly selected 3 or 4 objects from this set and presented them either in their original size $\left(4^{\circ} \times 4^{\circ}\right)$ or twice as large $\left(8^{\circ} \times 8^{\circ}\right)$. The objects were centered at a location $6^{\circ}$ away from fixation (similar to Figure 1).

Procedure and Design. Each trial started with the presentation of a fixation point for $500 \mathrm{msec}$, followed by the display of four objects, which was erased after $160 \mathrm{msec}$. The objects were either all unique, or the shape of two objects was the same. The two repeated objects were either identical or mismatched in size or viewpoint. Participants were asked to maintain fixation and look for repetitions in object shape while ignoring differences in size and viewpoint. Most objects were categorically different, but some were from the same basic category (e.g., two exemplars of chairs, which were considered to be different in shape).

Each participant completed nine blocks (Experiment 2A, 108 trials/block; Experiment 2B, 72 trials/block), separated by short breaks. In each block, half of the trials were unique trials and half were repeated trials. The size and viewpoint of each object was randomly determined. On repeated trials, the repeated objects could be identical (identical, one third of repeated trials), or the same in object shape and viewpoint but different in size (size mismatch, one third of repeated trials), or the same in shape and size but different in viewpoint (viewpoint mismatch, one third of repeated trials). In addition, the repeated objects occupied diagonal, bilateral, or unilateral positions equally often in Experiment 2A, and bilateral or unilateral positions equally often in Experiment 2B (no diagonal-repetition trials). The size and viewpoint of the unrepeated objects were randomly determined. Trial order was randomized within a block. Other aspects of the experiment were the same as in Experiment 1.

\section{Results and Discussion}

Accuracy (Figure 3 ) was similar across experiments (2A vs. $2 \mathrm{~B})(F<1)$, and there was no interaction between experiment and other factors (smallest $p=.20$ ). Accuracy was higher when the repeated objects were identical than when they differed in size or viewpoint, leading to a significant main effect of target type (identical, size mismatch, or viewpoint mismatch) $[F(2,32)=31.36, p<$ $.001]$. This effect verified that extracting shape invariance from size or viewpoint mismatched objects was costly (Vickery et al., 2005). There was also a significant UFA $[F(1,16)=47.19, p<.001]$. Notably, the UFA did not decrease when the repeated items differed in size [a $15.7 \%$ unilateral advantage; $t(17)=6.80, p<.001]$ or viewpoint [a $10.9 \%$ unilateral advantage; $t(17)=5.61, p<$ $.001]$ relative to when they were identical [a $10.3 \%$ unilateral advantage; $t(17)=4.54, p<.001]$. The interaction between target type and visual field was significant $[F(2,32)=3.82, p<.033]$, reflecting a larger UFA in the size-mismatch condition than in the other conditions.

Thus, the UFA was not smaller for repeated objects that differed in size or viewpoint, even though perceptual grouping should decrease and computational demands increase in those conditions. These results could not be accounted for by speed-accuracy trade-off (see the Appendix).

\section{EXPERIMENT 3}

The first two experiments manipulated perceptual grouping by reducing the similarity between repeated targets. In this experiment we reduced perceptual grouping by increasing the similarity between repeated and unrepeated items. Participants were shown four color patches and determined whether two of them were identical. The unrepeated colors were either distinctive or similar to the repeated colors. When all color patches were similar, perceptual grouping of the repeated colors was weakened and search became attentionally demanding (Duncan \& Humphreys, 1989). If perceptual grouping can fully account for the UFA, then it should be weakened when the color patches are similar.

\section{Method}

Participants. Eight participants (mean age, 26 years; 6 females) completed Experiment $3 \mathrm{~A}$ and 8 others (mean age, 23 years; 7 females) completed Experiment 3B. They all reported being righthanded.

Stimuli. We used 16 color patches, 4 from each of four color categories: red, green, blue, and yellow. The luminance and chromaticity of the color patches were: red $\left[60.9 \mathrm{~cd} / \mathrm{m}^{2}(.546, .365)\right]$, red $[62.9 \mathrm{~cd} /$ $\left.\mathrm{m}^{2}(.452, .361)\right]$, red $\left[50.5 \mathrm{~cd} / \mathrm{m}^{2}(.415, .364)\right]$, red $\left[28.8 \mathrm{~cd} / \mathrm{m}^{2}(.456\right.$ $.386)]$, green $\left[152 \mathrm{~cd} / \mathrm{m}^{2}(.301, .573)\right]$, green $\left[113 \mathrm{~cd} / \mathrm{m}^{2}(.307\right.$, $.509)]$, green $\left[78.5 \mathrm{~cd} / \mathrm{m}^{2}(.313, .475)\right]$, green $\left[47.7 \mathrm{~cd} / \mathrm{m}^{2}(.331\right.$, $.511)]$, blue $\left[35.7 \mathrm{~cd} / \mathrm{m}^{2}(.178, .118)\right]$, blue $\left[43.3 \mathrm{~cd} / \mathrm{m}^{2}(.212, .178)\right]$, blue $\left[38.0 \mathrm{~cd} / \mathrm{m}^{2}(.240, .222)\right]$, blue $\left[21.3 \mathrm{~cd} / \mathrm{m}^{2}(.252, .227)\right]$, yellow $\left[179.3 \mathrm{~cd} / \mathrm{m}^{2}(.402, .504)\right]$, yellow $\left[127 \mathrm{~cd} / \mathrm{m}^{2}(.384, .473)\right]$, yellow $\left[83.3 \mathrm{~cd} / \mathrm{m}^{2}(.376, .457)\right]$, and yellow [51.4 cd $\left./ \mathrm{m}^{2}(.394, .483)\right]$. 


\section{Experiment 2A}

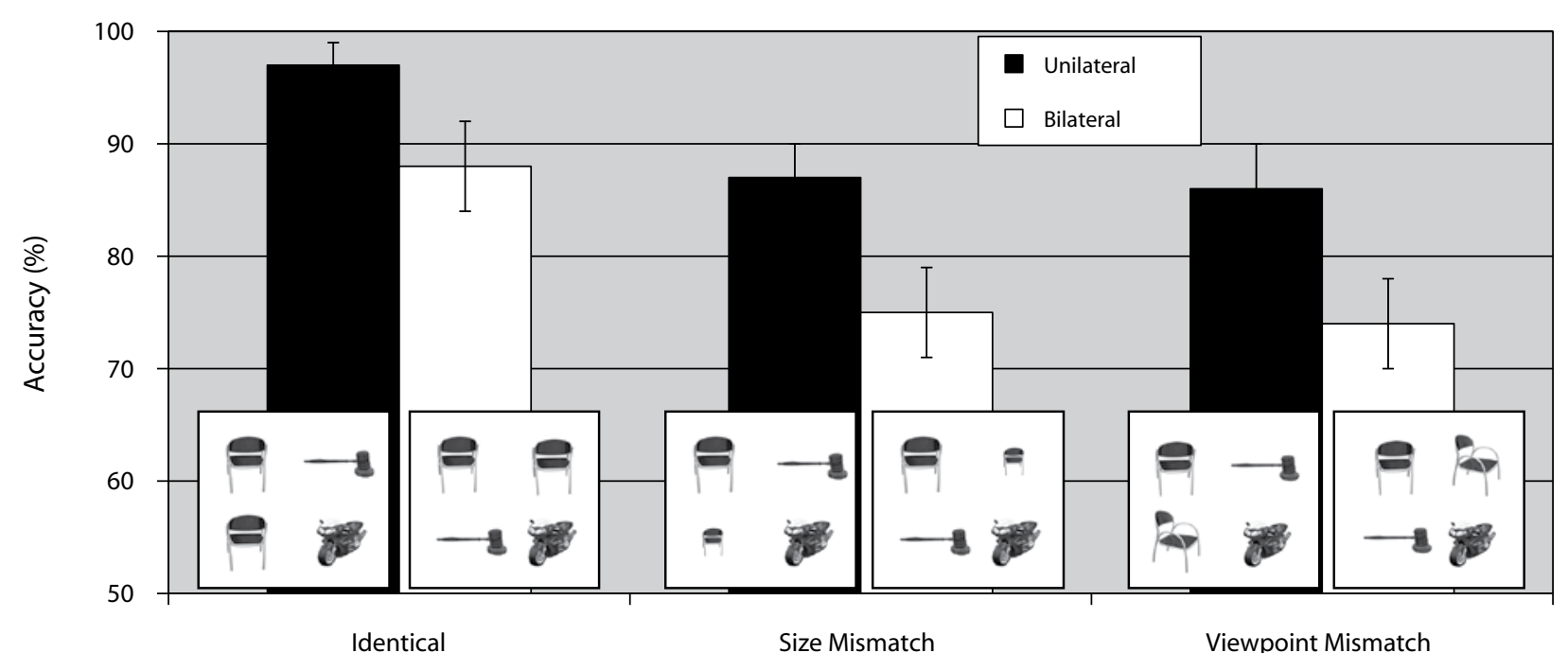

Repeated Objects

\section{Experiment 2B}

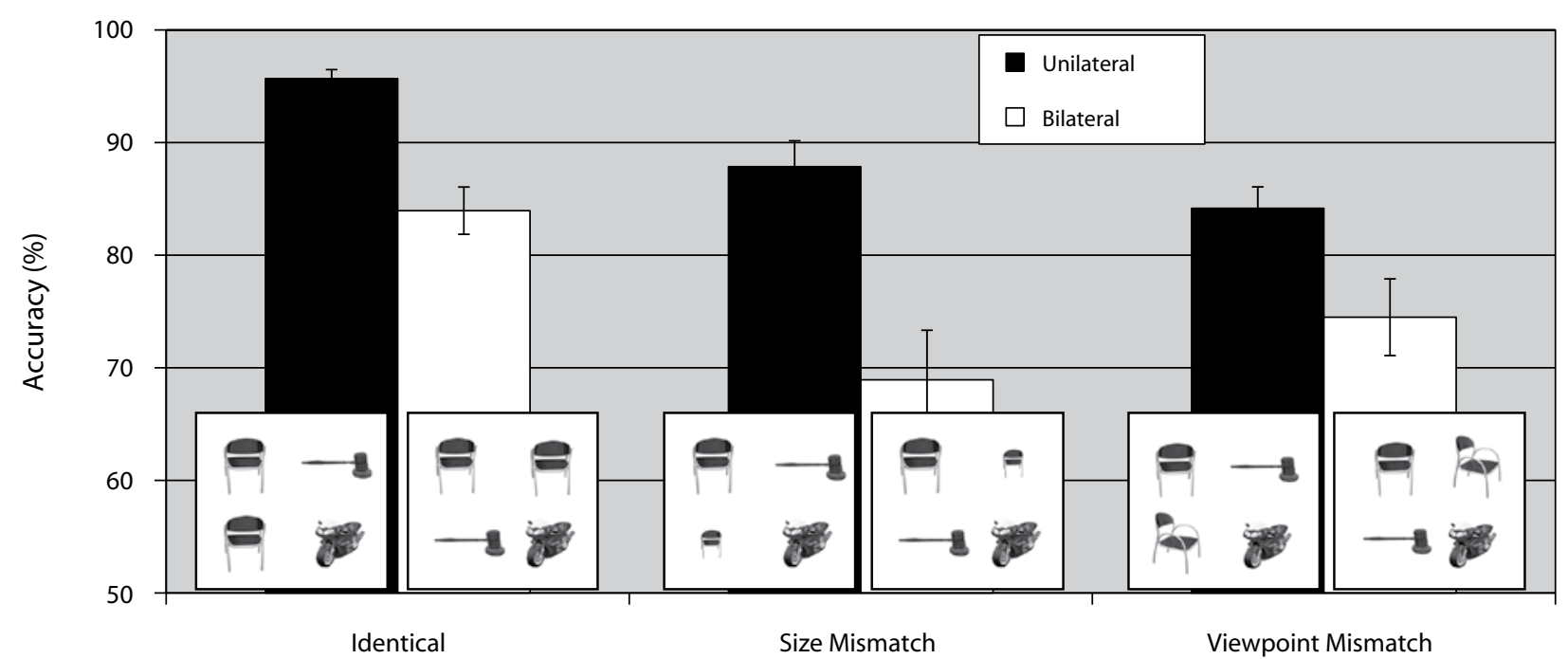

Repeated Objects

Figure 3. Results from Experiment 2. The illustrated conditions all received a "same" response (unique trials contained four different objects, not shown here). Error bars show $\pm 1 S E$ across participants.

Design and Procedure. Each participant was tested in 10 blocks (Experiment 3A, 96 trials/block; Experiment 3B, 64 trials/block). In each block, there were two types of trials, distinctive and similar. Colors on the distinctive trials were the most saturated color from each category, whereas colors on the similar trials were drawn from a single category. The four colors were all different from one another in unique trials, and two colors were the same in repeated trials. The repeated colors could occupy unilateral, bilateral, or diagonal positions equally often in Experiment $3 \mathrm{~A}$, and unilateral or bilateral positions equally often in Experiment 3B. On each trial, the display of four color patches was presented for $160 \mathrm{msec}$. All other aspects of the experiment were the same as in Experiment 1.

\section{Results and Discussion}

Accuracy (Figure 4) was unaffected by experiment (3A vs. 3B) or its interaction with the other factors (smallest $p=.25)$. We obtained a significant main effect of color similarity on accuracy $[F(1,14)=31.30, p<.001]$, with better performance in the distinctive than in the similar 
Experiment 3A

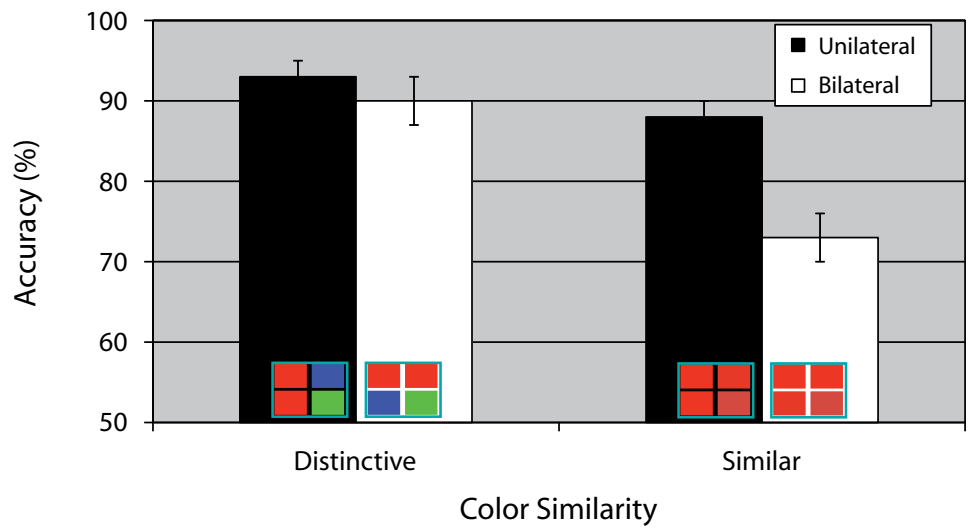

Experiment 3B

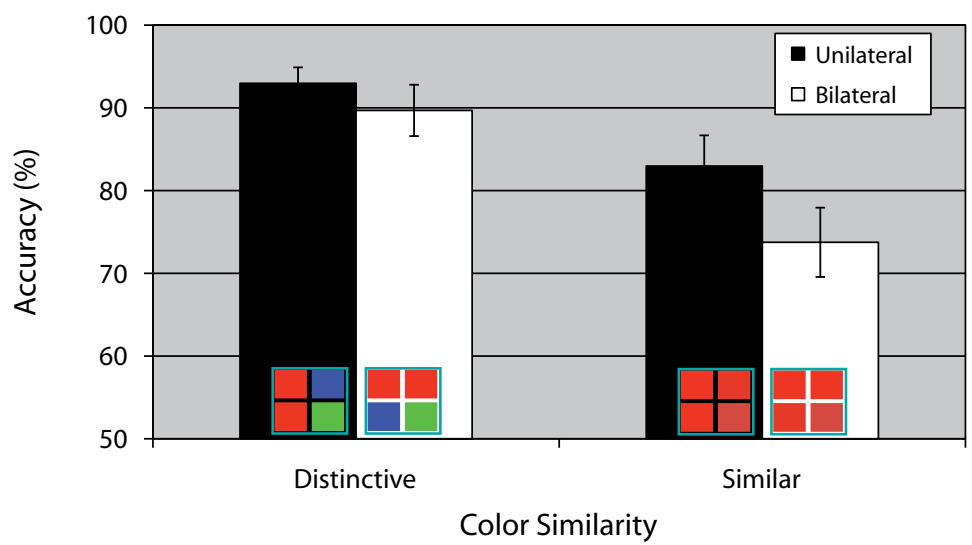

Figure 4. Results from Experiment 3. Error bars show $\pm 1 S E$ across participants.

condition. The main effect of visual field arrangement was significant, revealing a UFA $[F(1,14)=29.02, p<$ $.001]$. Critically, the UFA was larger when the colors were similar (a 10.4\% UFA) than when they were distinctive (a $4.7 \%$ UFA), resulting in a significant interaction between color similarity and visual field arrangement $[F(1,14)=$ $10.58, p<.006]$. RT data revealed no evidence for a speed-accuracy trade-off (see the Appendix).

\section{GENERAL DISCUSSION}

This study examined whether the UFA in repetition detection of simple visual stimuli can be fully accounted for by perceptual grouping strength of the repeated items, or the computational demands in the repetition-detection task. Data from three experiments presented a consistent pattern: Reducing perceptual grouping of the repeated items does not reduce the UFA; instead, the UFA either remains the same or becomes greater. The manipulations that we used to reduce perceptual grouping included using arrays of items that mismatched in color or letterform or in size or viewpoint, or that were highly similar. These ma- nipulations also increased task and attentional demands by requiring selective attention to a relevant stimulus property (MacLeod, 1991), requiring the transformation of objects' size or viewpoint (Peissig \& Tarr, 2007), or increasing target-distractor similarity (Duncan \& Humphreys, 1989). Nonetheless, increased task demands also failed to reduce the UFA.

One account that is largely compatible with the present data set is the callosal degradation account (Marsolek, Nicholas, \& Andresen, 2002). According to this account, the UFA results from the degradation of information that is transmitted across the corpus callosum. When precise perceptual information is needed for a task, callosal degradation should be particularly detrimental. Because perceptual comparison is needed in all conditions of our study, the callosal degradation account predicts that there should be a UFA in all conditions. In addition, the greater perceptual precision required by the similar colors condition of Experiment 3 should yield a larger UFA than the different colors condition. Our data are compatible with these predictions. However, the callosal degradation account cannot easily explain why the UFA is greater in the irrelevant- 
dimension mismatch condition of Experiment 1 and the size-mismatch condition of Experiment $2 .{ }^{3}$ Future research is needed to explore the source of increased UFA in these conditions. Regardless of the outcome of this research, however, the present experiments present significant constraints on simple predictions derived from the perceptual grouping and computation complexity accounts of repetition detection. Just as importantly, they highlight the need to further explore the nature of interhemispheric interactions in this task.

\section{AUTHOR NOTE}

This research was inspired by Serena Butcher's $\mathrm{PhD}$ thesis. It was supported by a University of Minnesota Grant in Aide to Y.V.J. and by an Undergraduate Research Opportunities Program grant to M.T.H. We thank Jen Decker and Bo-Yeong Won for data collection, and Patrick Cavanagh, Jillian Fecteau, Brad Gibson, Kalanit Grill-Spector, Tal Makovski, Chad Marsolek, Paige Scalf, Leah Watson, and two anonymous reviewers for comments and suggestions. Correspondence should be directed to K. M. Swallow or Y. V. Jiang, University of Minnesota, 75 East River Road, Minneapolis, MN 55455 (e-mail: khena.swallow@gmail.com or yuhong .jiang@gmail.com).

\section{REFERENCES}

BANICH, M. T., \& BELgER, A. (1990). Interhemispheric interaction: How do the hemispheres divide and conquer a task? Cortex, 26, 77-94.

Butcher, S. J., \& CaVanagh, P. (2008). A unilateral field advantage for detecting repeated elements. Perception \& Psychophysics, 70, 714-724.

Cavanagh, J. P., \& Parkman, J. M. (1972). Search processes for detecting repeated items in a visual display. Perception \& Psychophysics, 11, 43-45.

Duncan, J., \& Humphreys, G. W. (1989). Visual search and stimulus similarity. Psychological Review, 96, 433-458.

HunT, S. M. J. (1994). MacProbe: A Macintosh-based experimenter's workstation for the cognitive sciences. Behavior Research Methods, Instruments, \& Computers, 26, 345-351.

MACLEOD, C. M. (1991). Half a century of research on the Stroop effect: An integrative review. Psychological Bulletin, 109, 163-203.

Marsolek, C. J., Nicholas, C. D., \& Andresen, D. R. (2002). Interhemispheric communication of abstract and specific visual-form information. Neuropsychologia, 40, 1983-1999.

PeIsSIG, J. J., \& TARR, M. J. (2007). Visual object recognition: Do we know more now than we did 20 years ago? Annual Review of Psychology, 58, 75-96.

VicKerY, T. J., King, L. W., \& JiAnG, Y. (2005). Setting up the target template in visual search. Journal of Vision, 5, 81-92.

Weissman, D. H., \& Banich, M. T. (1999). Global-local interference modulated by communication between the hemispheres. Journal of Experimental Psychology: General, 128, 283-308.

Weissman, D. H., Banich, M. T., \& Puente, E. I. (2000). An unbalanced distribution of inputs across the hemispheres facilitates interhemispheric interaction. Journal of the International Neuropsychological Society, 6, 313-321.

\section{NOTES}

1. The UFA cannot be attributed to differences in processing horizontal and vertical arrays of objects. When presented unilaterally, repetition detection is equivalent for both configurations (Butcher \& Cavanagh, 2008).

2. Many previous studies used a three-item display, in which two different items are presented symmetrically at the top of the display, and a third to-be-compared item is presented at the bottom of the display, slightly lateralized to the left or right (Banich \& Belger, 1990). The three-item configuration yields a bilateral field advantage when the task requires semantic-level comparison. However, the three-item display does not match the item distance between the unilateral and bilateral trials and therefore is not used here (see also Butcher \& Cavanagh, 2008).

3. In Experiment 2, the UFA in the viewpoint-mismatch condition was not greater than the UFA in the identical condition, perhaps because some repetitions were detected on the basis of semantic repetition rather than visual perceptual repetition (Banich \& Belger, 1990). 
APPENDIX

Mean RTs (in Milliseconds) From Correct Trials in Unilateral Repetition and Bilateral Repetition Conditions

\begin{tabular}{|c|c|c|c|c|c|c|c|c|c|}
\hline \multirow[b]{2}{*}{ Experiment } & \multirow[b]{2}{*}{ Visual Field } & \multicolumn{2}{|c|}{$\begin{array}{c}\text { Color Task, } \\
\text { Letter } \\
\text { Match }\end{array}$} & \multicolumn{2}{|c|}{$\begin{array}{c}\text { Color Task, } \\
\text { Letter } \\
\text { Mismatch }\end{array}$} & \multicolumn{2}{|c|}{$\begin{array}{c}\text { Letter } \\
\text { Task, Color } \\
\text { Match } \\
\end{array}$} & \multicolumn{2}{|c|}{$\begin{array}{c}\text { Letter } \\
\text { Task, Color } \\
\text { Mismatch }\end{array}$} \\
\hline & & $M$ & $S E M$ & $M$ & SEM & $M$ & SEM & $M$ & $S E M$ \\
\hline \multirow[t]{3}{*}{$1 \mathrm{~A}$} & Bilateral & 606 & 41 & 642 & 61 & 768 & 53 & 998 & 46 \\
\hline & Unilateral & 548 & 41 & 580 & 36 & 674 & 48 & 866 & 44 \\
\hline & UFA & \multicolumn{2}{|c|}{$\begin{array}{l}58 \mathrm{msec} \\
p<.001\end{array}$} & \multicolumn{2}{|c|}{$\begin{array}{c}63 \mathrm{msec} \\
p<.10\end{array}$} & \multicolumn{2}{|c|}{$\begin{array}{l}94 \mathrm{msec} \\
p<.004\end{array}$} & \multicolumn{2}{|c|}{$\begin{array}{c}133 \mathrm{msec} \\
p<.01\end{array}$} \\
\hline \multirow[t]{5}{*}{$1 \mathrm{~B}$} & Bilateral & 648 & 56 & 701 & 59 & 812 & 75 & 903 & 83 \\
\hline & Unilateral & 628 & 54 & 706 & 73 & 769 & 81 & 887 & 80 \\
\hline & UFA & \multicolumn{2}{|c|}{$\begin{array}{c}20 \mathrm{msec} \\
p>.59\end{array}$} & \multicolumn{2}{|c|}{$\begin{array}{c}-5 \mathrm{msec} \\
p>.50\end{array}$} & \multicolumn{2}{|c|}{$\begin{array}{c}42 \mathrm{msec} \\
p>.43\end{array}$} & \multicolumn{2}{|c|}{$\begin{array}{l}17 \mathrm{msec} \\
p>.70\end{array}$} \\
\hline & & \multicolumn{2}{|c|}{$\begin{array}{c}\text { Identical } \\
\text { Objects }\end{array}$} & \multicolumn{2}{|c|}{$\begin{array}{c}\text { Size } \\
\text { Mismatch }\end{array}$} & \multicolumn{2}{|c|}{$\begin{array}{l}\text { Viewpoint } \\
\text { Mismatch }\end{array}$} & & \\
\hline & Visual Field & $M$ & $S E M$ & $M$ & SEM & $M$ & SEM & & \\
\hline \multirow[t]{3}{*}{$2 \mathrm{~A}$} & Bilateral & 776 & 68 & 874 & 63 & 877 & 60 & & \\
\hline & Unilateral & 728 & 70 & 843 & 79 & 809 & 67 & & \\
\hline & UFA & \multicolumn{2}{|c|}{$\begin{array}{c}48 \mathrm{msec} \\
p<.07\end{array}$} & \multicolumn{2}{|c|}{$\begin{array}{l}68 \mathrm{msec} \\
p<.007\end{array}$} & \multicolumn{2}{|c|}{$\begin{array}{c}30 \mathrm{msec} \\
p>.26\end{array}$} & & \\
\hline \multirow[t]{5}{*}{$2 \mathrm{~B}$} & Bilateral & 679 & 47 & 758 & 63 & 739 & 67 & & \\
\hline & Unilateral & 625 & 39 & 727 & 48 & 721 & 57 & & \\
\hline & UFA & \multicolumn{2}{|c|}{$\begin{array}{l}54 \mathrm{msec} \\
p<.06\end{array}$} & \multicolumn{2}{|c|}{$\begin{array}{c}31 \mathrm{msec} \\
p>.17\end{array}$} & \multicolumn{2}{|c|}{$\begin{array}{l}17 \mathrm{msec} \\
p>.40\end{array}$} & & \\
\hline & & \multicolumn{2}{|c|}{$\begin{array}{c}\text { Distinctive } \\
\text { Color }\end{array}$} & \multicolumn{2}{|c|}{$\begin{array}{c}\text { Similar } \\
\text { Color }\end{array}$} & & & & \\
\hline & Visual Field & $M$ & $S E M$ & $M$ & SEM & & & & \\
\hline \multirow[t]{3}{*}{$3 \mathrm{~A}$} & Bilateral & 654 & 32 & 764 & 45 & & & & \\
\hline & Unilateral & 619 & 34 & 707 & 34 & & & & \\
\hline & UFA & \multicolumn{2}{|c|}{$\begin{array}{c}34 \mathrm{msec} \\
p<.04\end{array}$} & \multicolumn{2}{|c|}{$\begin{array}{l}57 \mathrm{msec} \\
p<.06\end{array}$} & & & & \\
\hline $3 \mathrm{~B}$ & Bilateral & 720 & 65 & 872 & 84 & & & & \\
\hline & Unilateral & 679 & 50 & 834 & 82 & & & & \\
\hline & UFA & $\begin{array}{c}41 \\
p\end{array}$ & $\begin{array}{c}\text { asec, } \\
.06\end{array}$ & $\begin{array}{l}38 \\
p\end{array}$ & $\begin{array}{l}\text { isec, } \\
.05\end{array}$ & & & & \\
\hline
\end{tabular}

Note-In Experiment 1A, the UFA was stronger in the letter task than in the color task $(p<.024)$ but did not interact with other factors $(p \mathrm{~s}>30)$. In Experiment 1B, the UFA was insignificant overall $(p>.60)$ and did not interact with other factors $(p \mathrm{~s}>.10)$. In Experiment $2 \mathrm{~A}$, the UFA was significant overall $(p<.028)$ but did not interact with object conditions $(p>30)$. In Experiment $2 \mathrm{~B}$, the UFA was insignificant overall $(p>.20)$ and did not interact with object conditions $(p>.14)$. In Experiment $3 \mathrm{~A}$, the UFA was significant overall $(p<.014)$ but did not interact with similarity $(p>.45)$. In Experiment $3 \mathrm{~B}$, the UFA was significant overall $(p<.026)$ but did not interact with similarity $(p>.80)$. UFA, unilateral field advantage.

(Manuscript received September 15, 2009; revision accepted for publication October 24, 2009.) 